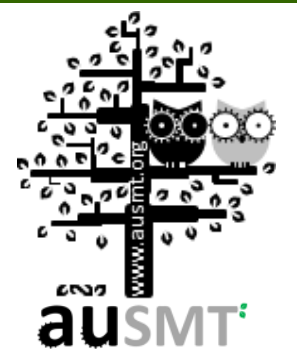

\title{
Innovation Effect on Patent Pool Formation: Empirical Case of Philips' Patents in Digital Versatile Disc 3C
}

\author{
Yu-Hui Wang 1, * and Benjamin Liu ${ }^{2}$ \\ ${ }^{1}$ Graduate Institute of Services and Technology Management, National Taipei University of Technology, Taiwan \\ ${ }^{2}$ The John Marshall Law School, USA \\ (Received 20 November 2012; Accepted 29 January 2013; Published on line 1 September 2013) \\ *Corresponding author: isecho@ntut.edu.tw \\ DOI: $10.5875 /$ ausmt.v3i3.176
}

\begin{abstract}
The aim of this paper is to understand the evolution of a patent pool over time, focusing on an individual firm. The legitimacy of patent pools requires a careful balance between innovation incentives, anti-competitive possibilities and consumer surpluses. Thus, it is an empirically important issue whether a patent pool continues to maintain this carefully struck balance even as the mix of its patent assets continues to evolve. The particular focus of this study is to examine the quality and quantity of Phillips' patent holdings within the DVD 3C patent pool in order to understand the extent of the firm's innovation within that patent pool over its lifetime, measured by four indicators that previous scholars have linked to the notion of patent quality. The result shows that, while the four indicators of patent quality varied, they together reveal that the breadth of exclusivity expanded significantly during the life of the patent pool, but that this expansion represented little innovative activity. Most of the patents issued post-formation are extensions of patents granted before pool-formation.
\end{abstract}

Keywords: Innovation; Patent Pool; DVD 3C; Patent Quality; Divisional Patent Application; Continuation Patent Application; Continuation-In-Part Patent Application

\section{Introduction}

Technology firms in an innovative industry may encounter overlapping or fragmented intellectual property rights owned by different companies that must be combined before commercialization can take place [1]. One solution to this problem is the formation of a patent pool where firms pool their patent assets together and license it as a package for the purpose of developing and commercializing technology-based products. When the patent pool combines complementary patents that are essential to the product, it enhances welfare by lowering the transaction cost of obtaining permission from the individual patent owners, avoiding the problem of double marginalization, and discouraging firms from engaging in patent hold-up. On the other hand, a patent pool can potentially create a technological cartel between licensors and reduce competition in the market and was historically disfavored by regulators until recently [2]. Governments now recognize that modern technological products often require the combination of many patents and are more willing to approve these patent pool arrangements [3].

In order to ensure that patent pools are used to bring together complementary technologies and to avoid the strategic use of patent pools to reduce competition and innovation between substitute technologies, antitrust regulators have been limiting patent pools by requiring them to bring together "essential patents" scattered among firms. Specifically, recently approved patent pools are often associated with "patents that are essential to compliance with Standard Specifications" [4], as exemplified by the MPEG-2 (Moving Picture Experts Group) patent pool that grew out of the MPEG-2 Video and Systems Coding standard or the DVD 3C (Digital Versatile Disc) and 6C patent pools that grew out of the DVD ROM and DVD Video standards (The DVD Forum) [5]. 
These pools require independent expert review to ensure that only essential patents are incorporated. These safeguards notwithstanding, even standard-based patent pools may generate some welfare reducing effect. One area of inquiry aims to understand to what extent patent pools reduce the incentive to innovate. Innovation, according to [6], is "carrying out new combinations" to generate inventions which may include "discovery scientifically new and can also exist in a new way of handling a commodity commercially." Patent pools can promote innovation by facilitating the recombination of new and existing patent technology across firms, a function that is critical for firm survival and growth in the high-tech sector [7, 8]. But recent empirical work suggests that patent pools may suppress further patenting and innovation in the technology area relating to the pool [9]. For example, licensors in the sewing machine patent pool, the earliest patent pool in the U.S., engaged in less innovation and patenting activities after the pool's formation [9]. For a more recently example, a similar tendency is observed for licensors in the optical disc related patent pools [10].

When regulators and scholars analyze the effect of patent pools, much of the analysis is based on a static portfolio model pegged at the time of pool creation, taking into account only the moments before and after pool formation. In actuality, the content of a patent pool continues to change after its formation, as new patents enter the pool. The possibility of an expanding pool introduces uncertainty about its effect on competition and innovation. For example, the United States Department of Justice (DOJ) patent pool review process provides an illustration of the potential mismatch that can occur when we overlook the temporal dimension. Philips held 40 patents in the Philips-Sony-Pioneer DVD patent pool as of 1998, the year when the DOJ approved the patent pool. However, new essential patents were added to the patent pool after its formation and the number of Philips patents eventually climbed to 99. This calls into question whether the balance of innovation, competition and consumer benefit used as

Yu-Hui Wang is an assistant professor at National Taipei University of Technology (NTUT), Taipei. She received her M.S. and Ph.D. degrees in Law from the National Chengchi University (NCCU) Taipei, Taiwan. Dr. Wang works cooperatively with faculties and researchers on many Intellectual Property Management projects. Dr. Wang currently also serves as editor of both the Journal of Communications and Information Sciences (JCIS) and Advances in Multimedia Technology (AMT). She also serves as co-director of the Intellectual Property and Industry Analysis Platform of NTUT.

Benjamin Liu is an assistant professor at The John Marshall Law School. Before joining academia, he represented Asian and American clients in patent litigation, prosecution and consulting. After receiving a bachelor's degree from Harvard University in biochemistry and cellular biology, Liu conducted drug discovery research at Eli Lilly \& Co. and later received his JD degree from UCLA School of Law. His research is in the area of international patent law, border IP enforcement, patent harmonization and innovation issues. the basis for including patents at the time of approval necessarily carries the same force forward if the size of the patent pool more than doubles. The pool expansion occurred throughout much of the product and technology cycle. When the DVD 3C license was first approved in 1998, it granted a 10 year license to DVD manufacturers. The patent pool continued to grow in the ensuing years until 2010, when the last patent was added to the pool. Thus for the rapidly developing high-tech industry with short product cycles, a patent pool should more accurately be understood as an evolving entity rather than a one-time event.

Several recent empirical examinations of patents pools studied pool features relating to pool management over time. [11] found that pools of complementary patents are more likely to allow independent licensing and require grant backs that can change the composition of the pool over time. [12] examine the rules by which revenues are allocated. Although their study primarily looks at the effect of allocation rules on the decision to participate in the pool, the same allocation rules may also affect firm behaviors that alter pool composition after its formation. The aim of this paper is to understand the evolution of a patent pool over time, focusing on an individual firm. The legitimacy of patent pools requires a careful balance between innovation incentives, anti-competitive possibilities and consumer surpluses. Thus, it is an empirically important issue whether a patent pool continues to maintain this carefully struck balance even as the mix of its patent assets continues to evolve. The particular focus of the study is to examine the quality and quantity of Phillips' patent holdings within the DVD $3 C$ patent pool to understand the firm's innovation within the patent pool over its lifespan.

\section{Hypothesis Development}

Firms in an industry with the greatest quantity and quality of patents are more likely to have their patents selected for inclusion in a patent pool. Therefore, they have strong incentives to increase the quantity and quality of their patent portfolio prior to the formation of the patent pool. This tendency is described as "portfolio racing" and has been empirically observed by [13]. A patent pool aggregates fragmented IP rights into a single package for licensees [14] and the innovation incentives of all of the firms in the industry change substantially subsequent to the pool formation [15]. After a patent pool is formed, the pool licensors generate less and lower quality patents in the technology field relating to the patent pool relative to those of nonparticipants [10].

There are two sets of explanations for the reduced innovative activity subsequent to the formation of the 
patent pool. First, licensors may wish to avoid unnecessary differentiation and competition and strive to preserve the stability of the patent pool instead [16]. Second, licensors may not wish to jeopardize the legitimacy of the pool by introducing later innovations that may run afoul of anti-trust regulators and incur the cost of litigation [17]. Together, these concerns discourage follow-on innovation within the patent pool.

As the general innovative activity decreases in the technology field, the number and quality of patents incorporated into the patent pool post-formation is expected to decrease as well. Based on these incentive changes, we hypothesize the following:

Hypothesis 1: Patent pool formation leads to fewer licensor patents being incorporated into the patent pool.

Hypothesis 2: Patent pool formation leads to lower quality licensor patents being incorporated into the patent pool.

It should be noted that patent quality is a complex notion and researchers have developed many different indicators to capture quality. This study employs four indicators that are particularly relevant to the quality of innovativeness-that is -the invention's contribution to the technological field: (1) number of independent claims; (2) citation count; (3) family status of the patent; and (4) a composite quality score generated by IPDSS, a patent evaluation service that generates a quality score based on a composite of ten indicators.

\section{Methodology}

\section{Firm Context}
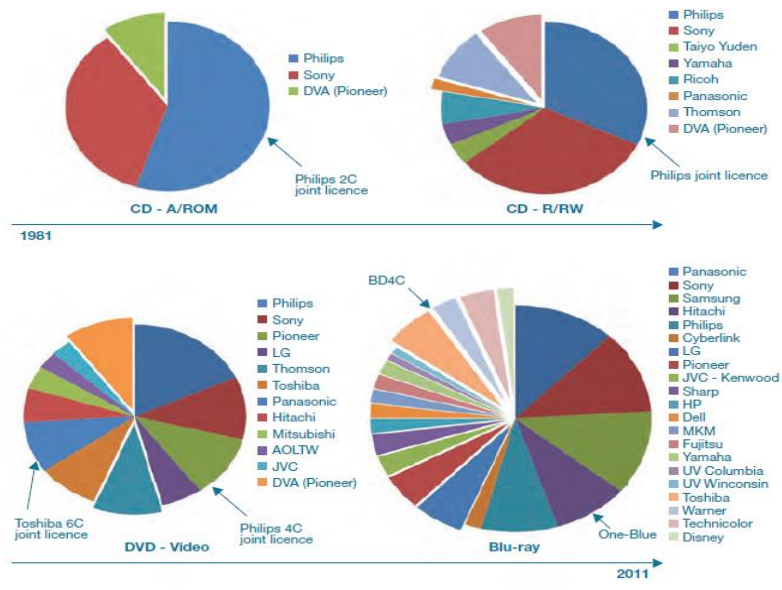

Figure 1. Number of licensors per optical standard [18].
The massive global optical disc industry is made possible by the systemic innovation of digital audio-visual technology and it represents a market in which multiple patent pools have been formed by competing firms [10]. The number of patents in the domain of optical discs and the number of companies that own these patents has risen sharply over the past 30 years as shown in Figure 1. The development of optical standards occured in 4 stages: CD-A/ROM in 1981, CD-R/RW, DVD-Video, and Blu-ray in 2011.

It is also worth taking a look at the historical impact of the patent pool on the DVD industry. It has been reported that DVD manufacturers must pay $\$ 15$ to $\$ 20$ in royalties for every new DVD player sold. Out of these royalties approximately $\$ 3.5$ to $\$ 5$ goes to the DVD 3C standard [19]. When companies avoid paying the hefty royalty fee, as Apex Digital did in 2001, SONY filed suit as a member of the DVD $3 \mathrm{C}$ pool and ultimately forced Apex to pay royalties [20]. Later, a Chinese DVD manufacturer, Wuxi Multimedia, Ltd., challenged the DVD 3C patent pool under antitrust law but was ultimately unsuccessful [21].

Philips, a technology-driven company, is a major participant in the optical industry and has invested billions of Euros on research and development, leading to new technologies and products. The fact that it is possible to obtain patents and licenses and repay part of the enormous cost of developing new and innovative products drives further research. This is why Philips aggressively initiated the formation of the MPEG-2, DVD $3 \mathrm{C}$ and One-Blue patent pools, and has been charged with the administration of DVD $3 C$

In order to examine Philips' patent holdings in the DVD 3 C patent pool, it was necessary to get a picture of the technology mix over time. To do this, we categorized the patents according to the International Patent Classification system (IPC) and the US Patent Classification (UPC) system. The IPC system was adopted by the World Intellectual Property Organization (WIPO) to provide for a hierarchical system of language independent symbols for the classification of patents. The UPC system organizes all U.S. patent documents and many other technical documents into relatively small collections based on common subject matter.

As shown in Figure 2, six patents belong to the IPC code G11B20/00 for "signal processing not specific to the method of recording or reproducing." Six other patents belong to G11B27/30 for "editing; indexing; addressing; timing or synchronizing; monitoring; measuring tape travel on the same track as the main recording." 


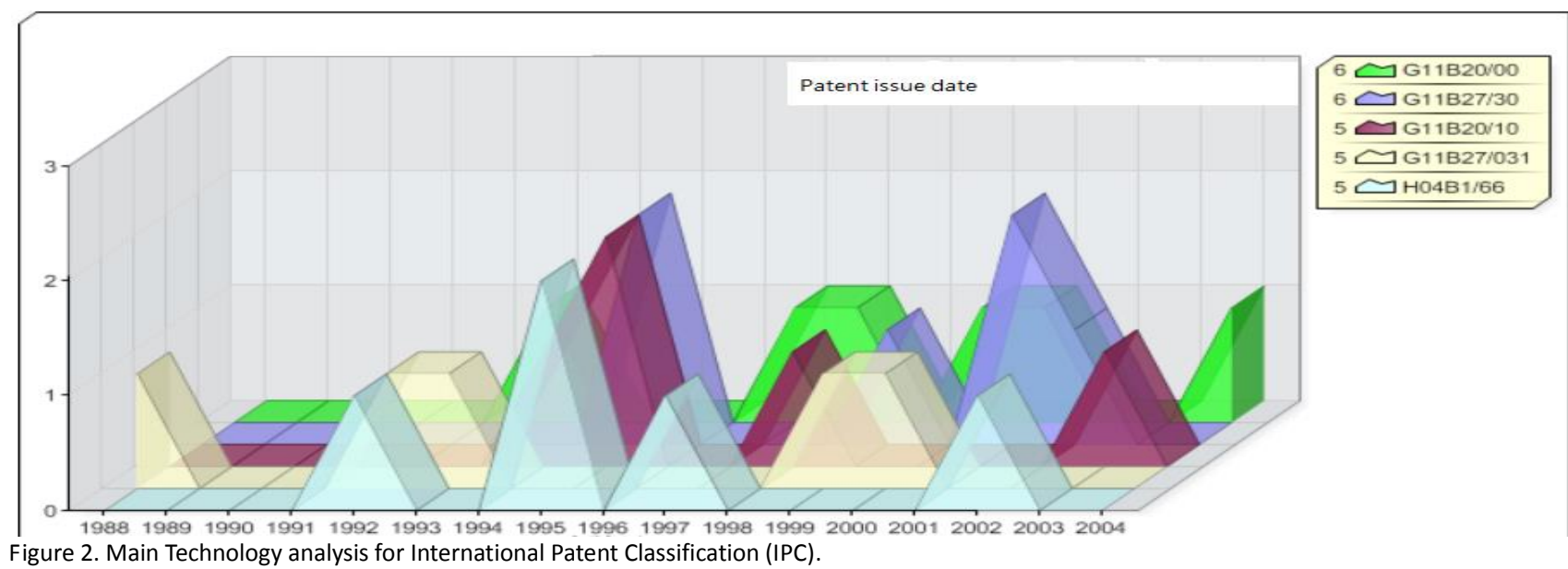

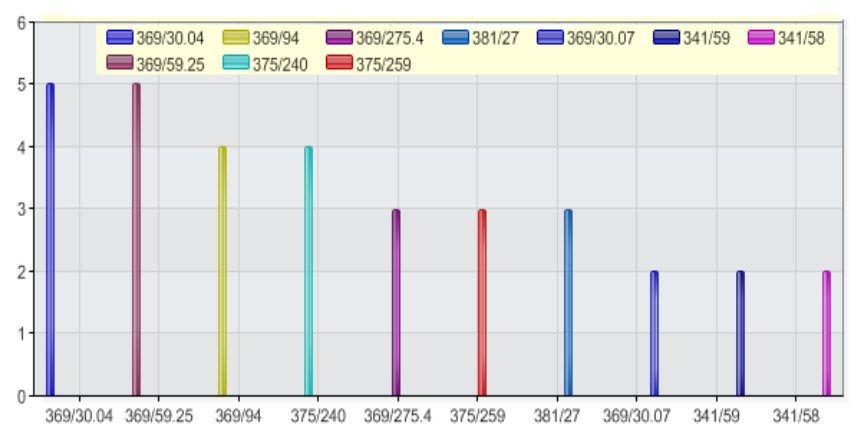

Figure 3. Main Technology Analysis for US Patent Classification (UPC).

As shown in Figure 3, the top technology categories according to the UPC code belong to UPC code $369 / 30.04$ and 369/59.25, defined as "dynamic information storage or retrieval using recorded information indicative of storage medium contents" and "dynamic information storage or retrieval format arrangement processing for auxiliary information" respectively. Each of these categories contains five patents.

\section{Sample construction}

We hypothesized that patent pool formation has a differential impact on the quantity and quality of innovation subsequent to the formation of the pool. In order to test the hypotheses associated with our proposed theory of patent pool formation and its impact on innovation incentives, we selected Philips as the subject of this case study because it is the most aggressive initiator of optical discs related patent pools. Specifically, we used Philip's patent holdings in the DVD $3 \mathrm{C}$ patent pool as the study sample. To test our hypotheses, we first collected all of the patents that were introduced by Philips and contributed to the DVD $3 C$ patent pool, using data provided by pool administrators (http://www.ip.philips.com/licensing/lp_programs.html). Of this set, we concentrated only on the patents granted in the United States totaling 99 patents. In the second step, we analyzed the information about these patents obtained through the U.S. Patent and Trademark Office (USPTO).

We analyzed the quantity and quality of the 99 patents which were filed from 1988 to 2006 . Because the pool was formed in 1998, we used the year 1998 (the year of the pool formation) as the critical year to differentiate the time period before and after patent pool formation.

\section{Variables of Interests}

In our study we examined patent statistics to assess the quantity and quality of innovation. This approach is consistent with the approaches taken in prior research studies which attempt to assess patent statistics as a proxy for the degree of innovation $[22,23]$.

To measure quantity, we counted the number of Philips' patents which were included in the DVD 3C patent pool annually between 1988 and 2006, according to filing year and issue year.

To measure patent quality, we chose four indicators: The number of independent claims, citation count, the patent family status as continuation, divisional, or continuation-in-part applications, and IPDSS score. Each of these indicators are described in detail below.

\section{The number of independent claims:}

The number of independent claims has long been used to measure patent quality. A large number of independent claims suggest greater innovative content, stronger exclusivity, and therefore a better quality patent [24].

\section{The citation count of a patent:}

Another measurement of quality is the citation count of a patent. Forward citation measures how often a patent has been cited by future patents and it is indicative of a high-impact innovation. Backward citation 
measures how many citations a patent has of others. A high number is correlated with an important research area and a broad innovation that is built on many prior works. Both types of citations have been positively linked to quality. Independent claim count, forward citation and backward citation have been combined to construct a patent quality index in the past [25].

\section{The family status of a patent:}

We used the family status of a patent as an additional test of the innovative quality of the patent based on whether it was based on a divisional patent application (hereinafter DA), a continuation patent application (hereinafter CA), or a continuation-in-part patent application (hereinafter CIP). In the Manual of Patent Examining Procedure (MPEP) § 201.06 which is published by the United States Patent and Trademark Office (USPTO), a DA is a later application for an independent and distinct invention carved out of an earlier filed copending application. In MPEP $\S$ 201.07, a CA is a second application for the same invention claimed in a prior copending nonprovisional application that claims the benefit of the filing date of the prior application. In 37 C.F.R. 153(d), a CA and DA must reference a previously filed application and must contain only matters disclosed in the previous application. Because CA and DA do not expand our knowledge, they do not represent quality innovation. In 37 C.F.R. 153(b), a $\mathrm{CIP}$ is an application filed during the lifetime of an earlier copending nonprovisional application, which claims the benefit of the filing date of the earlier application. However, a CIP repeats some substantial portion or all of the earlier application, but usually adds matter not disclosed in the earlier application and therefore represents a modest level of innovative quality.

\section{Composite quality scores generated by IPDSS:}

We used the Intellectual Property Defense-based Support System (IPDSS) to generate a report of Philips' patents quality. The system is an online patent analysis system (http://www.wheeljet.com.tw/). We downloaded the patents in the IPDSS and created a project by using the system to calculate the quality strength of a patent [26]. The indicators of patent quality of IPDSS were selected using principal components analysis (PCA) [27]. The PCA identified ten quality indicators, including patent examination time, numbers of US citations, numbers of foreign citations, science linkage, backward citations, patent families, technology cycle times, numbers of claims, numbers of independent claims, and UPCs. The summation of the ten indicators' score for every patent can be calculated and classified into 5 classes. A score of A or B indicates strong quality while a score of D or $E$ indicates weak quality.

\section{Data Analysis and Results}

The statistics results are reported in the following figures.

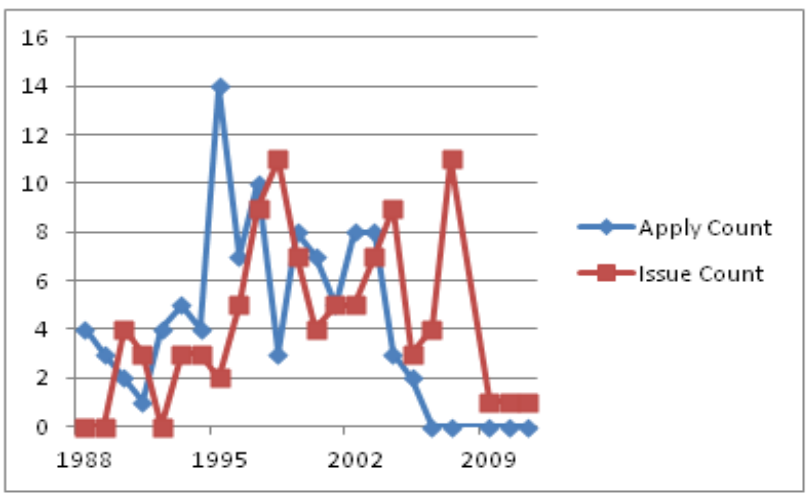

Figure 4. The statistics of patent filing and grant date.

Figure 4 shows the quantity of patents for every year from 1988 to 2011 . The diamond line is based on patent file date while the square line is based on patent grant date. To measure the timing of inventions, we record the grant dates and filing dates for all patents from 1988 to 2011. The peak period of patent filing application was from 1995 to 1997. 14 patents were filed in 1995. Fewer patent applications were filed in 1998 which was the critical year of pool formation, but the number remained elevated during 1999 to 2003.

The remaining data charts the trend relating to patent quality. For a more accurate analysis of activities in a given year, most results are based on the patent filing dates that are closer to the time of innovation.

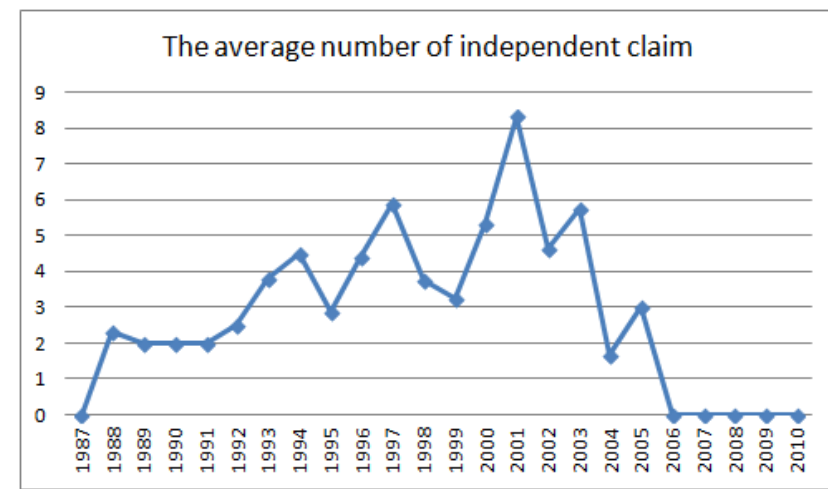

Figure 5 . The average number of independent claim of patents.

As shown in Figure 5, the average number of independent patent claims increased until 2002. The highest average number of independent claims was 8.33 in 2001. 


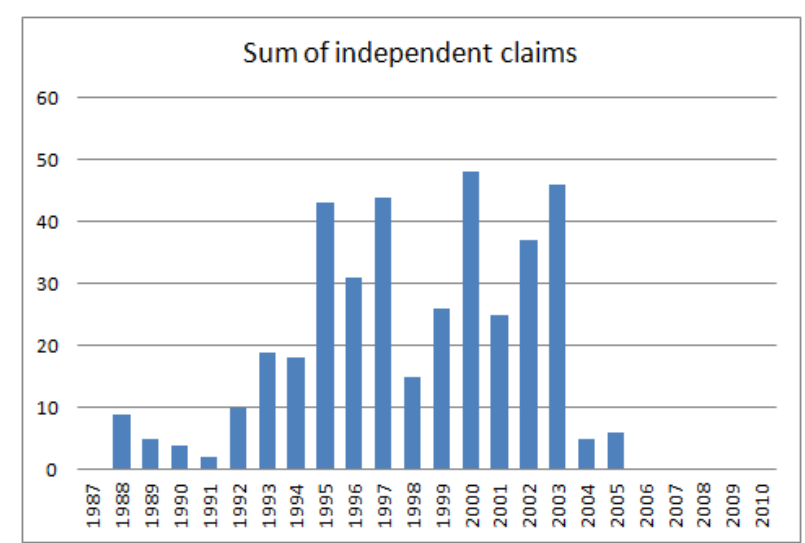

Figure 6. The sum of independent claim of patents.

The total number of independent claims granted a year is shown in Figure 6. 1995, 1997, 2000 and 2003 were peak years, having more than 40 granted independent claims for each of these years. 9 patents were filed in 2000 and together include 48 independent claims. A large number of independent claims (193) were granted after 1998, when the DVD 3C patent pool was formed.

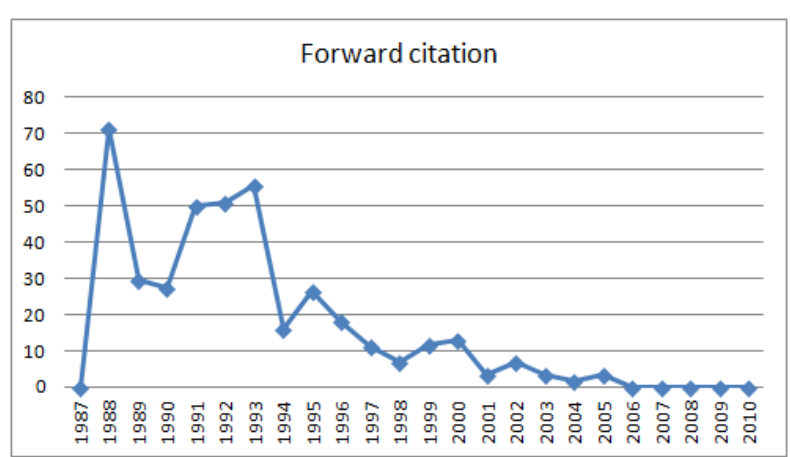

Figure 7. The average forward citations of patents.

The average number of forward citations per patent is shown in Figure 7. The average of forward citations of patents peaked early in 1988 at 71.3 and then generally decreased in subsequent years.

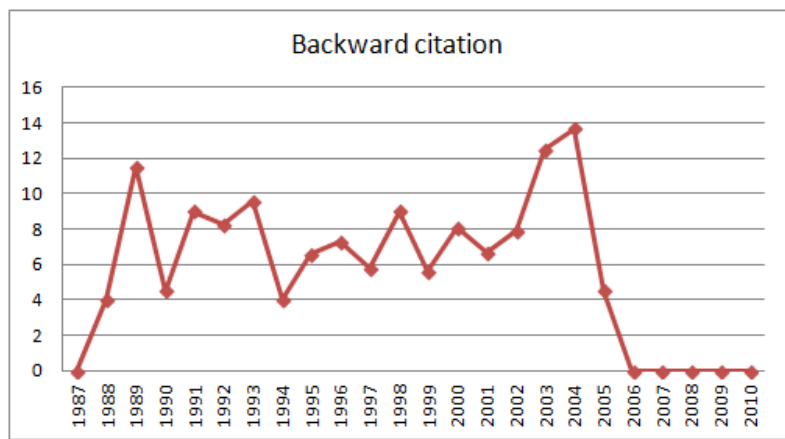

Figure 8 . The average of backward citations of patents.
As shown in Figure 8 , the average number of backward citations per patent peaked at 12.5 in 2003 and 13.67 in 2004. It hovered between 4 and 10 citations for much of the remaining years.

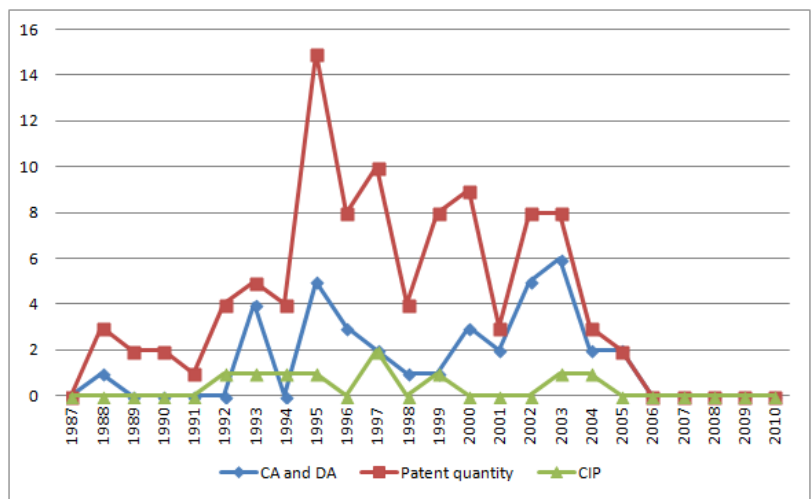

Figure 9. The number of patents, CA, DA and CIP applications.

Several peaks of CA, DA and CIP applications are shown in Figure 9. Five CA, DA and one CIP application were filed in 1995. The numbers of CA, DA and CIP applications are lowest in 1998 which was the critical year of pool formation. Afterward, the number of CA, DA applications remained raised until 2004.

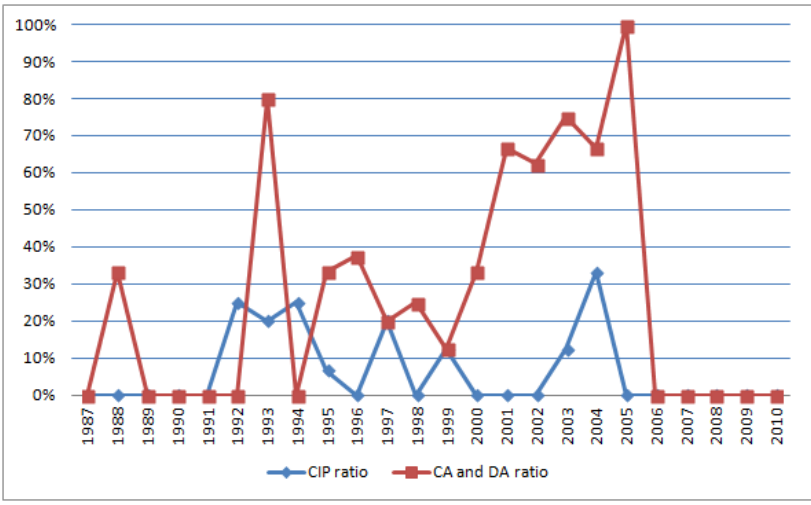

Figure 10. The ratio of CA, DA and CIP applications.

Figure 10 shows the ratio of CA, DA and CIP to the number of total Philip patents in a given year. There are a number of years when the ratio of CA and DA reaches over $60 \%$, indicating that most of the patents filed that year contained no new matter in their disclosure. The year 2005 shows a peak of $100 \%$ CA and DA patents. The ratio of CA, DA rose sharply from 2003 to 2006. It is notable that the ratios of CA, DA and CIP applications added up to 1 in 1992, 1993, 2004 and 2005 and it presents information showing that the total patents which were filed in these given years are CA, DA and CIP applications and that the years after 2001 generally show an elevated number of CA, DA and CIP applications. 


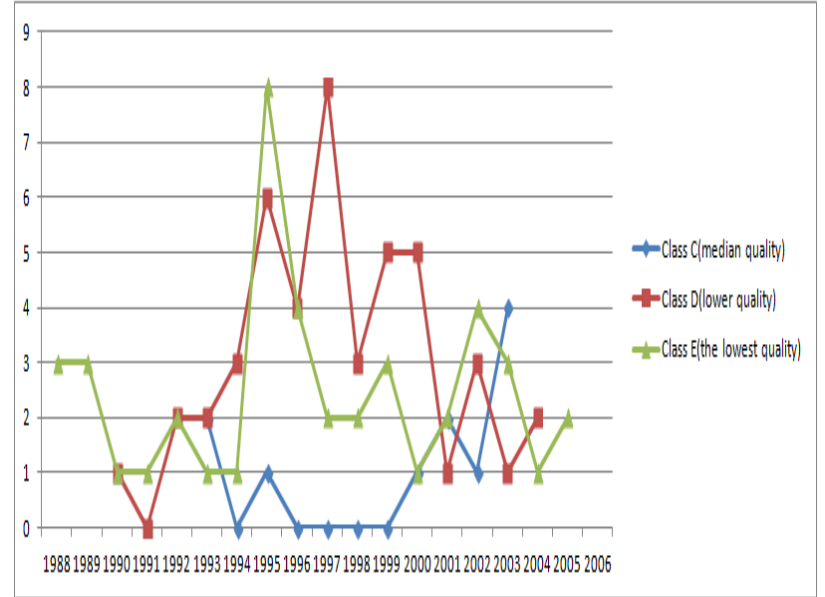

Figure 11. Composite quality score generated by IPDSS

From Figure 11, the IPDSS score identified 11 patents as median quality out of the 99 patents studied. 4 of them were filed in 2004 . The remaining majority received a low quality score.

\section{Discussion}

A close look at Philips' portion of the DVD 3 C patent pool reveals a continuously expanding patent pool with patent grants stretching over a long period of time. The first member of the Philip patent pool was granted in 1988 , ten years before the formation of the pool. 40 Philip pool patents were granted as of 1998, the year DOJ approval the pool. However, the patents issued after 1998 constituted the bulk of Philip's contribution to the patent pool, totaling 61 patents. The latest member for the patent pool was issued in 2011, 23 years after the first patent was issued and some of the earliest patents have already expired. Thus the patent pool was quite dynamic, capable of both expanding and contracting. This comes as a surprise, since the addition of patents does not increase royalty share and previous scholarship has noted several disincentives for additional investments in a standard technology. The dramatic expansion in the number of patents and claims confirms the need to examine patent pools periodically to ensure that, as more patents are added to a pool, that pool does not overreach and cover technological territories that should have been left open to competition. The expansion is even starker when we consider the growth of independent claims over time. The number of independent claims defines the scope of patent exclusivity and that number is being used as a proxy for the breadth of the technological advance, the rationale being that a patent with more independent claims sets up more fences within a technological space. In our sample, there were a total of 200 independent claims issued to patents filed as of 1998, and 193 independent claims issued to patents filed since.
Patent applications filed after the formation of the pool contain on average more independent claims than patent applications filed before the pool formation. Thus, the coverage of the patent pool significantly expanded since its creation. There are two alternative readings of this expansion vis-à-vis technological innovation. It is possible that, notwithstanding [10]'s finding of decreasing levels of innovation in areas relating to the patent pool, licensors continue to innovate within the actual patent pool itself in order to improve the technical features of the standard underlying the patent pool. Alternatively, the increasing patent numbers can represent an expansion of the patent pool exclusivity without advancing the field. In order to disentangle these possibilities, we turn to other quality measures to seek a fuller picture of the forces behind the pool expansion.

The divisional/continuation status is particularly revealing. Most applications filed after 1998 were continuation and divisional applications that contain information disclosures that are identical to patents that already exist in patents filed before the critical year of 1998. Although these patents stake out new legal exclusivity, they do not represent any new addition to the storehouse of knowledge. The small remaining portion of the post 1998 patent applications is comprised of CIP applications, which duplicates information in existing disclosures but also provides some additional innovation, albeit limited. Thus according to the historical data of the application family status, the quantity of patents and independent claims significantly expand the strength of the patent pool exclusivity without adding much innovation.

Other measures of patent quality are somewhat ambivalent. Backward citation analysis does not reveal any specific trend over the period studied but is consistent with the patent family analysis. Because much of the latter patents originated from continuation and divisional applications, the backward citation of these latter patents should mirror the backward citation of the earlier parent patent.

Forward citation analysis shows a general downward trend over time, consistent with the idea that an older patent is more likely to have been cited than a more recent patent, all else being equal. In order to eliminate the bias from patent age, we divided the average forward citation per patent for a given year by the number of years lapsed between the grant year of the patent and the present in order to arrive at an adjusted average. Figure 12 shows the annual forward citations per patent. The result shows that the average annual forward citation of the patents granted after the patent pool formation is still significantly lower than the patents that made up the initial patent pool, consistent 
with the idea that these post-formation patents are of a lesser innovation quality than their predecessors.

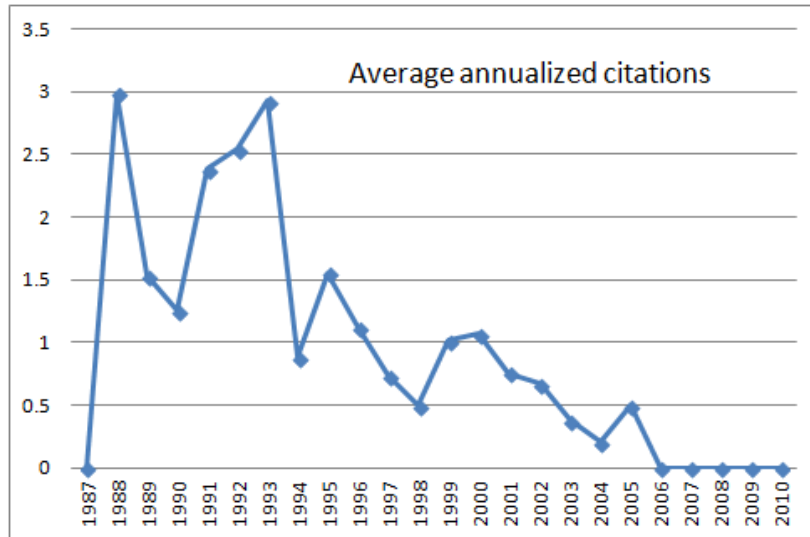

Figure 12. Adjusted average annualized citations.

Finally, none of the Phillip patents in the DVD 3C pool achieved a high quality score under the IPDSS system. Of the 99 total Philip patents, only 11 received a medium quality score and the remaining majority received a low quality score. These medium quality patents were issued at various points in time before and after the formation of the patent pool. It appears that, in light of the commercial importance of the early DVD patents, the IPDSS index under-estimates the innovation quality of the early patents issued prior to pool formation. Ironically, for patents issued after the formation that are largely derivative of earlier technology, the IPDSS index may have over-estimated their innovation quality. During the period before the patent pool formation, giving a mere medium score to 3 patents appears overly conservative and fails to capture the technological importance or the legal strength of these early "essential patents" that ultimately gave rise to the DVD industry and shaped the DVD standard. On the other hand, giving a medium score to 8 patents post 1998 appears overly generous and fails to capture their entirely derivative nature and the lack of innovative features. Ultimately the IPDSS composite scoring does not comport with the innovative characteristics of the patent pool.

\section{Conclusion}

This study examines how the portfolio of a patent pool changes over time by examining Philips' patents in the DVD 3C patent pool. The quality of innovation of the patents granted after the patent pool formation is lower than the patents that made up the initial patent pool, primarily because they merely regurgitated knowledge already disclosed in the earlier patents.

Interestingly, two indicators of patent quality, average annual forward citations of the patents and the family status of patents, support the hypothesis of patent pool formation leading to lower quality licensor patents being incorporated into the patent pool. In order to gain a fuller picture of the innovation effect on patent pool formation, we combined together the average number of independent claims with the family status of patents and concluded that the number of patents within a patent pool and the breadth of its exclusive rights can expand significantly subsequent to patent pool formation while the expanding patent pool represents very little additional innovation. Finally, none of the Phillip patents in the DVD 3 C pool achieved a high quality score under the IPDSS system. This shows that there is no significant difference in patent quality before and after pool formation. However, more information needs to be analyzed to figure out this kind of composite quality score. Our study also suggests the need of future studies that examine the entire portfolio included in the DVD 3C patent pool in order to develop a more detailed understanding of innovation effect on patent pools.

\section{Acknowledgment}

The authors thank the National Science Council of Taiwan for supporting this research under the grant of NSC 101-2221-E-027-080.

\section{References}

[1] G. Clarkson and D. Dekorte, "The problem of patent thickets in convergent technologies," Annals of the New York Academy of Science, vol. 1093, pp. 180-200, 2006.

[2] R. Gilbert and W. K. Tom, "Is innovation king at the antitrust agencies? The intellectual property guidelines five years later," ed: Industrial Organization 0106002, EconWPA, 2001.

Available:

http://www.escholarship.org/uc/item/8zv6b8c5

[3] P. B. De Laat, "Systemic innovation and the virtues of going virtual: The case of the digital video disc," Technology Analysis \& Strategic Management, vol. 11, no. 2, pp. 159-180, 1999. doi: $10.1080 / 095373299107483$

[4] The united states department of justice response to mr. Benny's inquiry (dvd 3c business review letter), 1998, [Online].

Available:

http://www.justice.gov/atr/public/busreview/2121 .pdf

[5] R. Bekkers, E. Iversen, and K. Blind, "Patent pools and non-assertion agreements: Coordination mechanisms for multi-party ipr holders in standardization," in the EASST Conference, Lausanne, Switzerland, 2006, pp.23-26. 
[6] J. A. Schumpeter and R. Opie, The theory of economic development; an inquiry into profits, capital, credit, interest, and the business cycle. Cambridge, Mass.: Harvard University Press, 1934.

[7] M. Boisot, Knowledge assets securing competitive advantage in the information economy, Oxford University Press, 1998, [Online].

Available:

http://search.ebscohost.com/login.aspx?direct=tru e\&scope $=$ site \&db=nlebk\&db=nlabk\&AN=56482

[8] S. G. Winter, "On coase, competence, and the corporation," Journal of Law, Economics, \& Organization, vol. 4, no. 1, pp. 163-180, 1988.

[9] R. L. Lampe and P. Moser, "Patent pools and the direction of innovation: Evidence from the 19th-century sewing machine industry," NBER Working Papers 17573, National Bureau of Economic Research, Inc, 2011.

[10] A. M. Joshi and A. Nerkar, "When do strategic alliances inhibit innovation by firms? Evidence from patent pools in the global optical disc industry," Strategic Management Journal, vol. 32, no. 11, pp. 1139-1160, 2011. doi: $10.1002 /$ smj.929

[11] J. Lerner, M. Strojwas, and J. Tirole, "The design of patent pools: The determinants of licensing rules," The RAND Journal of Economics, vol. 38, no. 3, pp. 610-625, 2007. doi: 10.1111/j.0741-6261.2007.00103.x

[12] A. Layne Farrar and J. Lerner, "To join or not to join: Examining patent pool participation and rent sharing rules," International Journal of Industrial Organization, vol. 29, no. 2, pp. 294-303, 2011. doi: 10.1016/j.ijindorg.2010.08.006

[13] B. H. Hall and R. H. Ziedonis, "The patent paradox revisited: An empirical study of patenting in the u.S. Semiconductor industry, 1979-1995," The RAND Journal of Economics, vol. 32, no. 1, pp. 101-128, 2001.

doi: $10.2307 / 2696400$

[14] R. Gilbert and C. Shapiro, "Optimal patent length and breadth," The RAND Journal of Economics, vol. 21, no. 1, pp. 106-112, 1990.

doi: $10.2307 / 2555497$

[15] J. Farrell and M. L. Katz, Innovation, rent extraction, and integration in systems markets. University of California at Berkeley: Institute of Business and Economic Research, 2000.

[16] G. M. Grossman and C. Shapiro, "Optimal dynamic r\&d projects," The Rand Journal of Economics, vol. 17, pp. 581-593, 1986.

Available: http://www.jstor.org/stable/2555483
[17] H. F. Chang, "Patent scope, antitrust policy, and cumulative innovation," in The Rand Journal of Economics vol. 26, ed: Spring, 1995, pp. 34-57.

[18] P. Ruud, "One-blue: A blueprint for patent pools in high-tech," Intellectual Asset Management, vol. 49, pp. 38-41, 2011.

[19] M. Clendenin, "Taiwan joins chinese effort on proprietary dvd format," EE Times, 2002.

Available:

http://eetimes.com/electronics-news/4043332/Tai wan-joins-Chinese-effort-on-proprietary-DVD-form at

[20] "China: Intellectual property rights," The Economist, 2005.

[21] S. M. Meisner and R. Lewis, "Antitrust claims against patent pool," IP update, vol. 11, no. 6, 2008.

[22] A. B. Jaffe and M. Trajtenberg, Patents, citations, and innovations a window on the knowledge economy, MIT Press, 2002, [Online].

Available:

http://search.ebscohost.com/login.aspx?direct=tru e\&scope $=$ site \&db=nlebk\&db=nlabk\&AN=70952

[23] A. J. C. Trappey, Y. H. Wang, T. H. Lin, and H. Y. Peng, "Empirical analysis of patent cross-licensing for light-emitting diode industry," presented at the 21th International Association for Management of Technology, Hsinchu, Taiwan, 2012.

[24] X. Tong and J. D. Frame, "Measuring national technological performance with patent claims data," Research Policy, vol. 23, no. 2, pp. 133-141, 1994. doi: 10.1016/0048-7333(94)90050-7

[25] J. O. Lanjouw and M. Schankerman, "Patent quality and research productivity: Measuring innovation with multiple indicators," The Economic Journal, vol. 114, no. 495, pp. 441-465, 2004. doi: 10.1111/j.1468-0297.2004.00216.x

[26] A. J. C. Trappey, C. V. Trappey, C.-Y. Wu, and C.-W. Lin, "A patent quality analysis for innovative technology and product development," Advanced Engineering Informatics, vol. 26, no. 1, pp. 26-34, 2012. doi: $\underline{10.1016 / j . a e i .2011 .06 .005}$

[27] H. F. Kaiser, "The application of electronic computers to factor analysis," Educational and Psychological Measurement, vol. 20, pp. 141-151, 1960.

doi: $\underline{10.1177 / 001316446002000116}$

